\title{
Precision Neuromuscular Block Management for Neural Monitoring During Thyroid Surgery
}

\author{
I-Cheng Lu, Sheng-Hua Wu, Pi-Ying Chang, Pi-Yang Ho, Tzu-Yen Huang, Yi-Chu Lin, Dipti V. Kamani, \\ Gregory W. Randolph, Gianlorenzo Dionigi, Feng-Yu Chiang, and Che-Wei Wu
}

\section{QUERY SHEET}

This page lists questions we have about your paper. The numbers displayed at left are hyperlinked to the location of the query in your paper.

The title and author names are listed on this sheet as they will be published, both on your paper and on the Table of Contents. Please review and ensure the information is correct and advise us if any changes need to be made. In addition, please review your paper as a whole for typographical and essential corrections.

Your PDF proof has been enabled so that you can comment on the proof directly using Adobe Acrobat. For further information on marking corrections using Acrobat, please visit http://journalauthors.tandf.co.uk/production/acrobat.asp; https://authorservices.taylorandfrancis.com/how-to-correct-proofs-with-adobe/

The CrossRef database (www.crossref.org/) has been used to validate the references.

\section{AUTHOR QUERIES}

Q1 Please check if the affiliations have been set correctly.

Q2 Please check if the affiliations have been set correctly.

Q3 Please provide the volume number.

Q4 Please provide the volume number.

Q5 Please provide the volume number.

Q6 Please provide the volume number and page range.

Q7 Please note that the ORCID section has been created from information supplied with your manuscript submission/CATS. Please correct if this is inaccurate. 


\section{Precision Neuromuscular Block Management for Neural Monitoring During Thyroid Surgery}

I-Cheng Lu, ${ }^{a, b}$, Sheng-Hua Wu ${ }^{a, b}$, Pi-Ying Chang ${ }^{a}$, Pi-Yang Ho ${ }^{a}$, Tzu-Yen Huang ${ }^{c}$, Yi-Chu Lin ${ }^{c}$, Dipti V. Kamani ${ }^{d}$, QI Gregory W. Randolph ${ }^{\text {d,e }}$, Gianlorenzo Dionigi ${ }^{f}$ (D) Feng-Yu Chiang ${ }^{g}$, and Che-Wei Wu ${ }^{b, c}$

${ }^{a}$ Department of Anesthesiology, Kaohsiung Municipal Siaogang Hospital, Kaohsiung Medical University, Kaohsiung, Taiwan; ${ }^{b}$ Faculty of Medicine, College of Medicine, Kaohsiung Medical University, Kaohsiung, Taiwan; 'Department of Otorhinolaryngology, Kaohsiung Medical University Hospital, Kaohsiung, Taiwan; ${ }^{d}$ Division of Thyroid and Parathyroid Endocrine Surgery, Department of Otolaryngology,

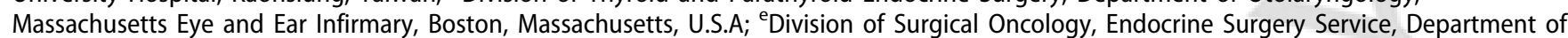
Surgery, Massachusetts General Hospital, Harvard Medical School, Boston, Massachusetts, U.S.A; 'Division for Endocrine Surgery, Department of Human Pathology in Adulthood and Child-hood "G. Barresi", University Hospital G. Martino, University of Messina, Messina, Sicily, Italy; ${ }^{9}$ Department of Otolaryngology, E-Da Hospital, I-Shou University, Kaohsiung, Taiwan

\begin{abstract}
Introduction: Titration of neuromuscular block (NMB) plays a key role in intraoperative recurrent laryngeal nerve monitoring during thyroid surgery. The combination of neuromuscular blocking agent and timely partial reversal of NMB was investigated in both animal experiments and clinical neuro-monitored thyroidectomy.

Methods: In animal experiments, 8 piglets received sugammadex to assess the laryngeal EMG recovery after rocuronium-induced NMB. In clinical monitored thyroidectomy, 40 patients each were allocated to conventional group and sugammadex group. Conventional group received rocuronium $0.3 \mathrm{mg} / \mathrm{kg}$ at anesthesia induction, while sugammadex group received partial NMB recovery protocol- $0.6 \mathrm{mg} / \mathrm{kg}$ of rocuronium at anesthesia induction and $0.5 \mathrm{mg} / \mathrm{kg}$ of sugammadex. Main outcome was assessed by first (V1) and final (V2) EMG signal induced by vagal stimulation.

Results: In the porcine model, 50\% recovery of laryngeal EMG amplitude was achieved at $16.8 \pm 1.9$ and $6 \pm 2.7$ minutes respectively after 0.5 and $1 \mathrm{mg} / \mathrm{kg}$ of sugammadex $(p<0.01)$. In monitored thyroidectomy, EMG amplitudes at V1 in group S and group C were $1214 \pm 623$ and $915 \pm 476 \mu \mathrm{V}$, respectively $(p=0.02)$. Positive and adequately high EMG amplitudes were observed at the early surgical stage for all patients. Sugammadex groups were superior to conventional group in EMG tube placement $(p<0.001)$.

Conclusion: Both porcine model and clinical application showed that precise NMB management by low-dose sugammadex was effective for intraoperative neural monitoring (IONM). The regimen ensured optimal conditions for tracheal intubation and timely neuromuscular function restoration for high-quality EMG signal.
\end{abstract}

\section{ARTICLE HISTORY}

Received 20 May 2020

Revised 30 June 2020

Accepted 28 July 2020

\section{KEYWORDS}

Thyroid surgery; intraoperative neural monitoring (IONM); recurrent laryngeal nerve (RLN); neuromuscular blocking agent (NMBA); sugammadex; anesthesia

\section{Introduction}

Nerve injury to recurrent laryngeal nerve (RLN) and/or external branches of superior laryngeal nerve (EBSLN) are one of serious complications related to the thyroid and parathyroid surgery. In recent decades, thyroid surgeons are devoted to reduce these complications by intraoperative neural monitoring (IONM) system. IONM could be used to map and identify the RLN and EBSLN, to detect nerve anatomic variations, to elucidate injury mechanisms and to predicting the prognosis of invaded or injured RLN to modify the surgical plans. ${ }^{1-11}$

Neuromuscular blocking agents (NMBA) are generally accepted as routine anesthesia practice for tracheal intubation and neck surgery. The proper administration of an NMBA is a key element of anesthesia to ensure successful IONM. ${ }^{12-16}$ To minimize the effect of NMBA on reduction of EMG signal and subsequent interpretation, sugammadex 95 could be used to timely and effectively reverse rocuronium- 97 induced neuromuscular block (NMB). Sugammadex as an 98 antagonist to steroidal NMBA is applied in patients requir- 99 ing IONM during thyroid or parotid surgery. ${ }^{17-19}$

There is a controversy between routine and selective 101 reversal of NMB with sugammadex in monitored thyroidec- 102 tomy. In our previous study, we confirmed a routine 103 fully-NMB-reversal protocol with sugammadex $(2 \mathrm{mg} / \mathrm{kg}) 104$ provided both excellent intubation condition and high initial 105 EMG signal of IONM in $100 \%$ of 50 patients. ${ }^{17}$ In contrast, 106 Empis et al. reported a selective sugammadex-reversal proto- 107 col and showed that sugammadex is required only 15108 (12.5\%) of 120 patients to enhance the first (V1) EMG sig- 109 nal induced by vagal stimulation $\left(\mathrm{V}_{1}\right) \cdot{ }^{18}$ However, the select- 110 ive protocol may result in a false-negative interpretation and 111 


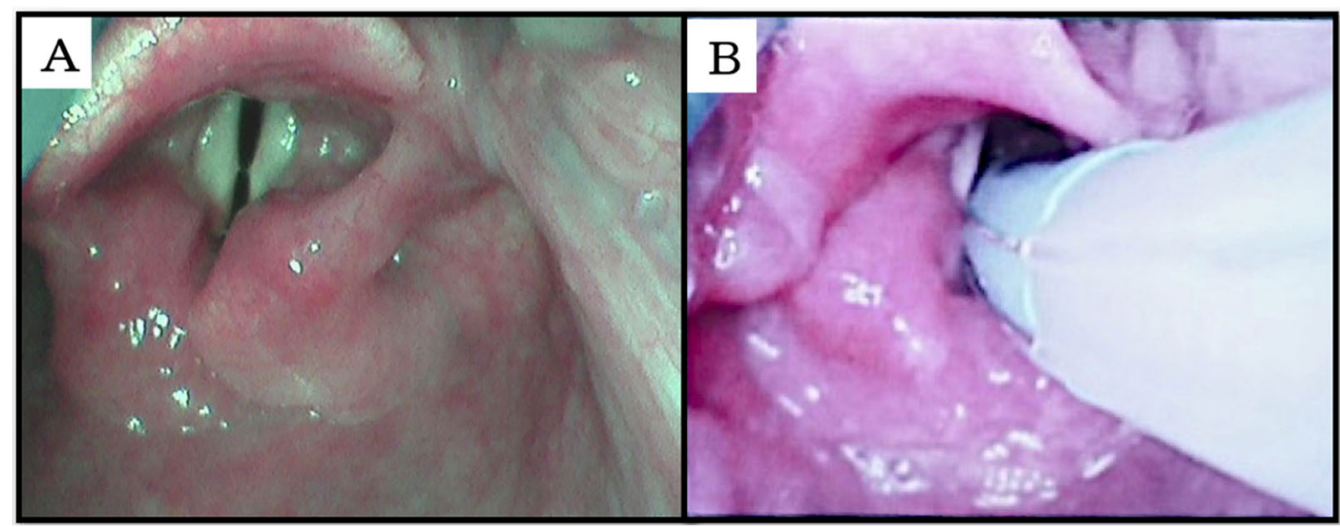

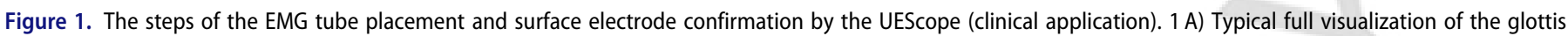
in a patient. 1B) Two pairs of surface electrodes are advanced perpendicular to the vocal cords until they reach the proper depth.

was measured at 3 time points: 1) detectable signal, 2) 50\% of baseline, and 3) $80 \%$ of baseline value.

\section{Clinical application}

We reviewed medical records of 80 patients (61 women; age from 24 to 80 years old) undergoing total thyroidectomy or total lobectomy under routine IONM assistance from Jan 2017 to Dec 2018. The Institutional Review Board of XXXX University Hospital approved this clinical trial (KMUH-IRB$\mathrm{E}(\mathrm{I})-20190164)$. All 80 patients were cared by single surgeon and experienced anesthesiologists. The IONM equipment setup, procedures and loss of signal algorithm followed the International Neural Monitoring Study Group Guidelines. ${ }^{1-4}$

General anesthesia induction was done by lidocaine $(2 \mathrm{mg} / \mathrm{kg})$, fentanyl $(1 \mu \mathrm{g} / \mathrm{kg})$ and propofol $(2 \mathrm{mg} / \mathrm{kg})$ for each patient. Patients were segregated into control group (Group C, $n=40$ ) if they received conventional anesthesia protocol with rocuronium of one effective dose $(0.3 \mathrm{mg} / \mathrm{kg})$ for anesthesia induction. In the sugammadex group (Group $S, n=40$ ), patients had received precision anesthesia protocol with rocuronium of standard dose $(0.6 \mathrm{mg} / \mathrm{kg})$ for anesthesia induction and low sugammadex dose $(0.5 \mathrm{mg} / \mathrm{kg})$ was administered intravenously 10 minutes after skin incision, (Table 1). All patients were subjected to a standard neural monitoring anesthesia protocol for thyroidectomy except NMBA regimen.

A reinforced EMG endotracheal tube (internal diameter (ID) $6.0 \mathrm{~mm}$ for female and $7.0 \mathrm{~mm}$ for male patients, respectively) (Medtronic, Jacksonville, FL) was placed by the UEScope (UE Medical Devices, Newton, MA). The EMG tube was then advanced under video guidance until the surface electrodes were in optimal contact with vocal cords (Figure 1). Proper tube depth and invisible tube rotation were then visually verified by video image and anesthesia was maintained with sevoflurane combined with propofol target-controlled infusion. The precision anesthesia protocol (Group S) included anesthesia depth and NMB management: At 10 minutes after operation starting, an intravenous bolus of sugammadex $0.5 \mathrm{mg} / \mathrm{kg}$ was administrated to partially reverse NMB (Figure 2). No additional rocuronium was given intraoperatively to any patient. Train-of-four

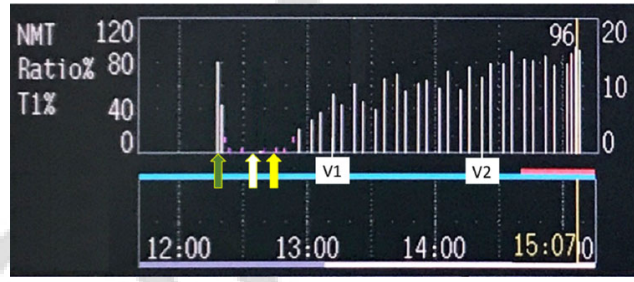

Figure 2. Typical time sequence of intraoperative neuromuscular transmission (NMT) monitoring in a patient with precision anesthesia protocol (clinical application). Green arrow: At anesthesia induction, the NMT was intact and baseline TOF ratio was around $100 \%$. White arrow: At skin incision, complete neuromuscular blockade was noted. The TOF response showed none of NMT and first twitch (T1) were $0 \%$. Yellow arrow: At 10 minutes after skin incision, sugammadex was given and NMT recovered gradually. $\mathrm{V} 1=$ first vagal stimulation. $\mathrm{V} 2=$ final vagal stimulation.

307

308

309

color

Beyly in

Print

312

313

314

315

316

317

318

319

320

321

(TOF) ratio derived via neuromuscular transmission (NMT) 322 monitor (Aestiva/5; Datex-Ohmeda, FL) was used to con- 323 tinuously assess NMB degree of the adductor pollicis muscle. 324 The train of four (TOF) stimulation was setup as (constant 325 $50 \mathrm{~mA}$ current, four twitches at every 0.5 second over 326 2 seconds). Anesthesia depth was monitored by Bispectral 327 index (BIS) monitor (Medtronic, MN). Target anesthesia 328 depth was controlled via titration by inhaled sevoflurane to 329 keep Bispectral index (BIS) monitor between 40\% and 50\%. 330

All thyroid surgery followed standard IONM proce- 331 dures, ${ }^{27,28}$ and the highest EMG amplitudes were captured, 332 registered, and compared. $V_{1}$ and $V_{2}$ signal was defined as 333 first vagal stimulation before thyroid dissection and final 334 vagal stimulation after thyroid resection, respectively $\quad 335$

Main outcome was assessed by $V_{1}$ and $V_{2}$ amplitude 336 $(\mu \mathrm{V})$ between groups; time course of hemodynamic parame- 337 ters and degree of NMB were also compared; while other 338 outcomes included physical characteristics, adverse events 340 and postoperative surgical outcomes. Extubation time was 341 defined as time interval from skin closure to EMG tube 342 removal. Each patient received laryngofiberoscopic examin- 343 ation to obtain vocal cord mobility recordings at the day 344 before and after surgery. When abnormal vocal cord move- 345 ment was noticed after operation, the recording before oper- 346 ation was used to compare.

To ensure adequate power for the study, the minimal 348 sample size was 20 patients in each group according to pre- 349 vious study. ${ }^{29}$ We reviewed records of 40 patients to show 350 

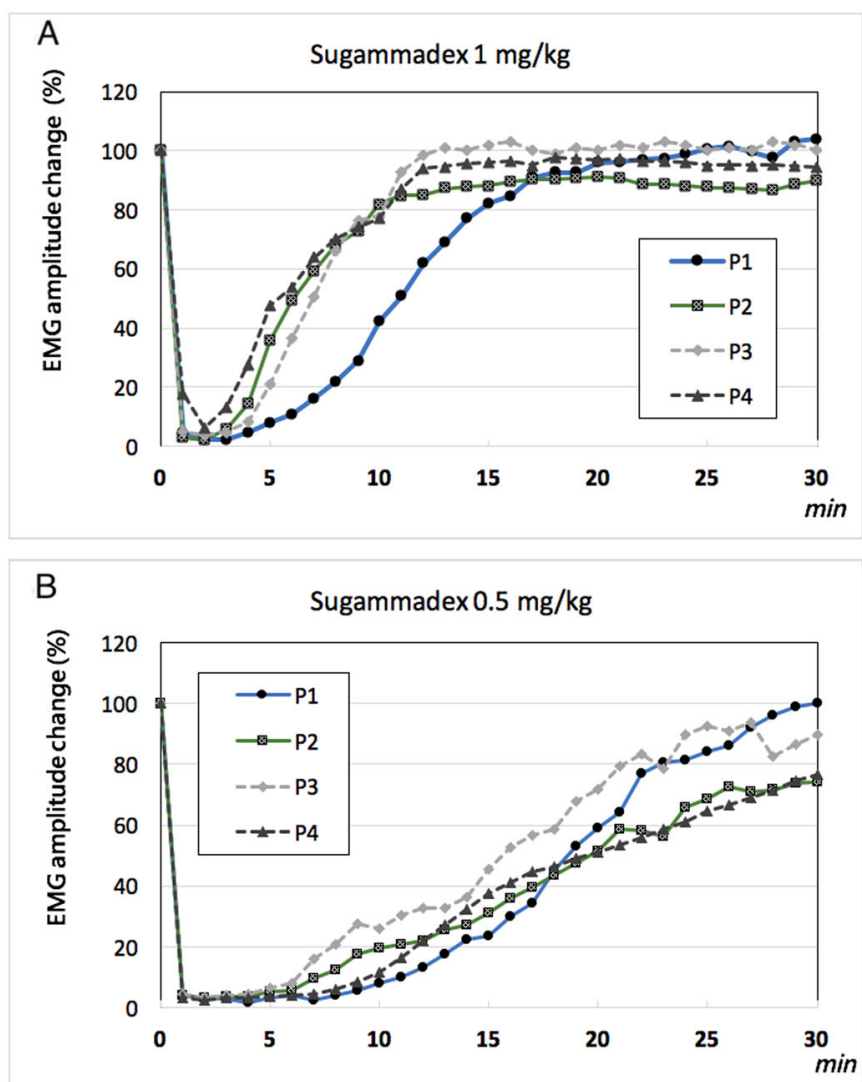

Figure 3. Tracing of laryngeal EMG amplitude of partial neuromuscular blockade reversal in each piglet (porcine model). Piglets received an intravenous bolus of rocuronium $0.6 \mathrm{mg} / \mathrm{kg}$ to induce complete neuromuscular block. Two minutes later, a bolus of sugammadex $1 \mathrm{mg} / \mathrm{kg}(3 \mathrm{~A})$ or $0.5 \mathrm{mg} / \mathrm{kg}(3 \mathrm{~B})$ was randomly injected. The observation period for laryngeal EMG recovery was 30 minutes.

at least $20 \%$ difference in EMG amplitude with a power of 0.9 and a type 1 error of 0.05 . All data are presented as mean (standard deviation). Statistical analysis of continuous variables between two groups was carried out by the 2 -sample $t$-test. Categorical variables were carried out by the chisquare test or the Fisher exact test. All statistical analysis was 2 -tailed, and a $\mathrm{p}<0.05$ was of statistical significance.

\section{Results}

In porcine model, rocuronium $0.6 \mathrm{mg} / \mathrm{kg}$ suppressed the EMG signal in all 8 piglets within a minute. Figure 3 shows tracing of laryngeal EMG amplitude of partial NMB reversal by low-dose sugammadex in individual piglets. It took $2.3 \pm 1.0$ and $6.5 \pm 2.3$ minutes to obtain detectable EMG signals (amplitude $>100_{\mu} \mathrm{V}$ ) after sugammadex 1.0 and $0.5 \mathrm{mg} /$ $\mathrm{kg}$ respectively $(\mathrm{p}=0.005)$. Sugammadex $1 \mathrm{mg} / \mathrm{kg}$ showed significantly faster EMG recovery to $50 \%(6.0 \pm 2.7$ vs. $16.8 \pm 1.9 \mathrm{~min})$ and $80 \%(9.8 \pm 2.2$ vs. $24.3 \pm 3.3 \mathrm{~min})$ baseline value than did sugammadex $0.5 \mathrm{mg} / \mathrm{kg}$ (both $\mathrm{p}<0.01$ ). In this porcine model, no significant changes in arterial blood pressure, heart rate and EKG rhythm were noted during vagal nerve stimulation and sugammadex administration. There was also no sign of residual blockade with observation at 30 minutes.
In the clinical trial, detailed characteristics of 80 patients are shown in Table 2. There were 137 nerves at risks, and we encountered one temporary and one permanent RLN palsy in two thyroid cancer patients. Most patients experienced excellent and good intubation quality and all EMG tubes placement were successful at the first attempt. Patients in group $S$ (rocuronium $0.6 \mathrm{mg} / \mathrm{kg}$ during induction, and sugammadex $0.5 \mathrm{mg} / \mathrm{kg} 10$ minutes after skin incision) had better intubation condition than those in group $\mathrm{C}$ (rocuronium $0.3 \mathrm{mg} / \mathrm{kg}$ during induction only) $(\mathrm{p}=0.002)$.

Time interval from anesthesia induction (rocuronium injection) to $\mathrm{V}_{1}$ and $\mathrm{V}_{2}$ stimulation did not differ significantly between groups as shown in Table 3 . It took an average of $18.5 \pm 10.2$ minutes from sugammadex injection to $V_{1}$ stimulation in group S. Figure 2 demonstrates typical time sequence of intraoperative neuromuscular transmission in a patient with partial reversal of NMB. Time course of precision anesthesia protocol including neuromuscular transmission and anesthesia depth during monitored thyroidectomy is depicted in Figure 4.

Neuromuscular blockage degree and EMG amplitude were compared at $\mathrm{V}_{1}$ and $\mathrm{V}_{2}$ stimulations between the two groups. At $\mathrm{V}_{1}$ stimulation, patients in group $\mathrm{S}$ showed higher EMG amplitude $(1214 \pm 623 \mu \mathrm{V}$ vs. $915 \pm 476 \mu \mathrm{V}$, $\mathrm{p}=0.02)$ and Train-of-four (TOF) ratio $(58.7 \pm 17.1$ vs. $44.9 \pm 20.1, \mathrm{p}=0.002$ ) than group $\mathrm{C}$ patients (Table 3). Patients in group $\mathrm{S}$ showed comparable EMG amplitude and TOF ratio as group C patients at V2 stimulation (both $p>0.05)$. Extubation time did not differ significantly between the two groups (Table 3). None of the patients had clinical event due to recurrent or residual NMB after sugammadex injection.

\section{Discussion}

In this porcine model, $0.6 \mathrm{mg} / \mathrm{kg}$ of rocuronium dose was used to induce a complete NMB. Both $1 \mathrm{mg} / \mathrm{kg}$ and $0.5 \mathrm{mg} /$ $\mathrm{kg}$ sugammadex doses showed at least approximate $80 \%$ laryngeal EMG amplitude recovery within a 30-minute observation period. It took an average of 6 and 17 minutes to achieve $50 \%$ EMG amplitude recovery by sugammadex 1 and $0.5 \mathrm{mg} / \mathrm{kg}$, respectively. Similarly, the clinical application shows the precision neuromuscular management protocol with low-dose sugammadex $(0.5 \mathrm{mg} / \mathrm{kg})$ provided timely and high-quality laryngeal EMG signal (mean amplitude $1214 \mu \mathrm{V}$ after average of 18.5 minutes from sugammadex injection). It facilitated better intubation condition, faster neuromuscular recovery and higher EMG signals compared to conventional anesthesia protocol (single one effective rocuronium dose at anesthesia induction). To the best of our knowledge, this is the first study using low-dose sugammadex $(0.5 \mathrm{mg} /$ $\mathrm{kg}$ ) to obtain partially reversal of NMB during IONM in thyroid surgery.

Many anesthesia protocols have been developed to minimize the interference of anesthesia on the IONM system during thyroid surgery. The degree of NMB is one of the major anesthesia factors that has been investigated over decades. If $\mathrm{NMB}$ degree is not titrated for IONM, EMG 
Table 2. Patient characteristics of 80 patients receiving monitored thyroidectomy with conventional and low-dose sugammadex protocols (clinical application).

\begin{tabular}{|c|c|c|c|}
\hline & Sugammadex (S Group, $n=40$ ) & Conventional (C Group, $n=40$ ) & $P$ value \\
\hline Female: Male (n) & 30:10 & $31: 9$ & 0.79 \\
\hline Age $(y r)$ & $50.0 \pm 11.5$ & $50.6 \pm 13.9$ & 0.82 \\
\hline Weight (kg) & $64.9 \pm 13.4$ & $62.9 \pm 14.7$ & 0.51 \\
\hline Height (cm) & $161.4 \pm 8.0$ & $161.1 \pm 7.5$ & 0.86 \\
\hline BMI $\left(\mathrm{kg} / \mathrm{m}^{2}\right)$ & $24.8 \pm 3.9$ & $24.0 \pm 4.1$ & 0.36 \\
\hline Intubation condition & & & $<0.001$ \\
\hline Excellent/good/poor & $23 / 17 / 0$ & $12 / 26 / 2$ & \\
\hline Vasopressor* (n) & $2(5 \%)$ & $5(12.5 \%)$ & 0.23 \\
\hline Benign/Cancer & $26 / 14$ & $30 / 10$ & 0.32 \\
\hline Nerve at risk $(\mathrm{n})$ & 72 & 65 & \\
\hline Temporary palsy (n) & $0(0 \%)$ & $1(1.5 \%)$ & 0.34 \\
\hline Permanent palsy $(\mathrm{n})$ & $0(0 \%)$ & $1(1.5 \%)$ & 0.34 \\
\hline Post-op hematoma (n) & $0(0 \%)$ & $0(0 \%)$ & 1.0 \\
\hline $\begin{array}{l}\mathrm{BMI}=\text { body mass index. } \\
\mathrm{S} \text { group (rocuronium } 0.6 \\
0.3 \mathrm{mg} / \mathrm{kg} \text { during indu }\end{array}$ & ring induction, and sugammadex & 10 minutes after skin incision), C & uronium \\
\hline
\end{tabular}

Table 3. A comparison of neuromuscular blockade degree, neural monitoring recordings and postoperative adverse events (clinical application).

\begin{tabular}{lcc}
\hline & Sugammadex (S Group, $n=40)$ & Conventional $(C$ group, $n=40)$ \\
\hline$V_{1}$ stimulation & & \\
Time from anesthesia (minutes) & $44.6 \pm 11.1$ & $42.1 \pm 13.9$ \\
EMG amplitude $(\mu \mathrm{V})$ & $1214 \pm 623$ & $915 \pm 476$ \\
TOF ratio (\%) & $58.7 \pm 17.1$ & $44.9 \pm 20.1$ \\
V2 stimulation & & 0.39 \\
Time from anesthesia (minutes) & $83.2 \pm 17.0$ & $76.3 \pm 18.2$ \\
EMG amplitude $(\mu \mathrm{V})$ & $1299 \pm 651$ & $1103 \pm 438$ \\
TOF ratio (\%) & $85.2 \pm 13.9$ & $83.9 \pm 12.2$ \\
Extubation time & $4.7 \pm 11.5$ & 0.02 \\
\hline
\end{tabular}

EMG $=$ electromyography, $\mathrm{TOF}=$ train of four, $\mathrm{V}_{1}=$ initial vagal stimulation.

$\mathrm{V}_{2}=$ final vagal stimulation, $\mathrm{S}$ group (rocuronium $0.6 \mathrm{mg} / \mathrm{kg}$ during induction, and sugammadex $0.5 \mathrm{mg} / \mathrm{kg} 10$ minutes after skin incision),

$\mathrm{C}$ group (rocuronium $0.3 \mathrm{mg} / \mathrm{kg}$ during induction only)..

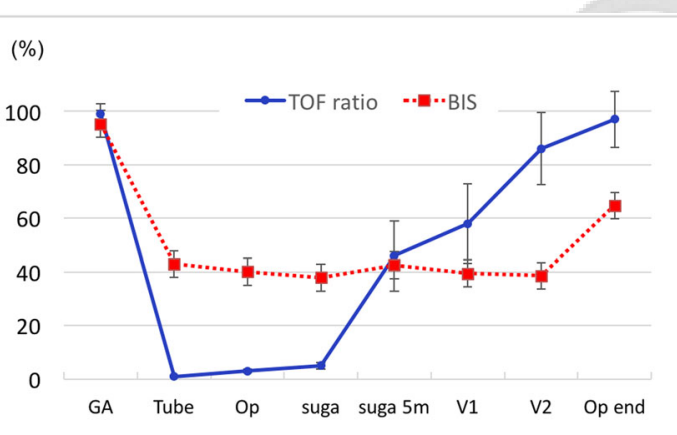

Figure 4. Time course of precision anesthesia protocol including neuromuscular transmission and anesthesia depth (clinical application). Neuromuscular transmission was monitored by train-of-four (TOF) ratio and anesthesia depth was assessed by bispectral index (BIS) derived from electroencephalogram (EEG).

$\mathrm{GA}=$ General anesthesia induction

Tube $=$ Tracheal intubation of EMG tube

Op $=$ Operation beginning

Suga $=$ Intravenous injection sugammadex $0.5 \mathrm{mg} / \mathrm{kg}$ at 10 minutes after operation

Suga $5 \mathrm{~m}=$ Five minutes after sugammadex injection

$\mathrm{V} 1=$ initial vagal stimulation (44.6 $\pm 11.1 \mathrm{~min}$ from anesthesia)

$\mathrm{V} 2=$ final vagal stimulation $(83.2 \pm 17.0 \mathrm{~min}$ from anesthesia)

amplitude might be undetectable or markedly low when nerve stimulation is performed during surgical steps. ${ }^{29,30}$

Several feasible NMB regimens for monitored thyroidectomy have been proposed. The goals of optimal NMB for monitored thyroidectomy should provide not only excellent relaxation for tracheal intubation and surgical procedure but also rapid recovery of neuromuscular transmission for neural monitoring when nerve stimulation is tested. The combination of rocuronium and sugammadex is one of the solutions to ensure intubation condition, surgical relaxation and timely neuromuscular function recovery. ${ }^{12,17,18} 562$ Sugammadex is a modified $\gamma$-cyclodextrin developed to 563 selectively bind amino-steroidal NMBAs (i.e., rocuronium) 564 but not isoquinoline NMBAs (i.e., cisatracurium). ${ }^{31-33} 565$ Sugammadex reverses rocuronium-induced NMB more 566 effectively than cholinesterase inhibitors (i.e., neostigmine); 567 hence, it archives faster extubation after surgery and less 568 postoperative residual curarization. ${ }^{34}$

Sugammadex dosage to prevent postoperative residual curarization is well defined according to peripheral nerve response (TOF ratio). During monitored parotid or thyroid surgery, a dose of $2 \mathrm{mg} / \mathrm{kg}$ sugammadex could induce nearly complete NMB recovery within 10 minutes. ${ }^{17,19}$ Since muscle 574 relaxation is also required during surgery, complete neuro- 576 muscular function recovery may not be the best manage- 577 ment protocol. The main purpose of this study was to 578 investigate suitable dosage and timing of sugammadex 579 administration. We confirmed that low-dose sugammadex 580 $(0.5 \mathrm{mg} / \mathrm{kg})$ was effective for obtain an excellent EMG 581 response at the first vagus nerve stimulation (V1) in both 582 porcine model and clinical application. Mean time required 583 to obtain $50 \%$ recovery of EMG signal in piglets was 584 16.8 minutes. In the human surgical setting, the timing of 585 sugammadex administration was designed at 10 minutes 586 
after skin incision. On an average, initial vagal stimulation $\mathrm{V}_{1}$ was obtained at 28.5 minutes after skin incision. This duration ranged variously from 11 to 55 minutes. Our hospital served as a referral center and received patients with higher surgical difficulty such cancer, large goiter or repeated surgery. Those surgical steps required much more time than common thyroid surgery. The protocol showed that partial recovery of neuromuscular function (averaged TOF $>58 \%$ ) was feasible for high-quality $V_{1}$ EMG signal (mean amplitude $1214 \mu \mathrm{V}$ with standard waveform) during monitored thyroidectomy.

Vagus nerve stimulation and obtain an initially satisfactory $\mathrm{V}_{1}$ baseline EMG signal is a mandatory part for accurate neural monitoring during thyroid surgery. ${ }^{1,3,10,28,35}$ Few studies have been proposed to apply sugammadex in IONM to avoid interference by NMB. Empis and coworkers proposed to use sugammadex in limited circumstances, they used it in only $12.5 \%(15 / 120)$ of patients whose EMG amplitude was less than $100{ }_{\mu} \mathrm{V}$ at $\mathrm{V} 1$ stimulation. ${ }^{18}$ This selective neuromuscular recovery protocol could meet the neural monitoring demand and be more cost-effective than routine sugammadex use. From the surgeon's perspective, a higher EMG signal is generally requested for better decision-making; however, a notable weakness of the protocol was that all patients in general had relatively low V1 signal with medium and lowest EMG amplitude being 402.5 and $214.5{ }_{\mu} \mathrm{V}$ respectively. Medium and lowest EMG amplitudes were 522 and $180{ }_{\mu} \mathrm{V}$ for obese population respectively. Conversely, when routine sugammadex was used, EMG amplitude of $V_{1}$ signal was much higher and stable at a mean value above $1,200{ }_{\mu} \mathrm{V}$ in this study and in a previous report. ${ }^{17}$

To compare cost-effectiveness between sugammadex and neostigmine, two issues about neural monitoring and residual neuromuscular block were addressed. To facilitate intraoperative neural monitoring, sugammadex is superior to neostigmine. In our results, sugammadex even in smaller dose could restore adequate neuromuscular transmission to obtain high-quality EMG signal. Conversely, neostigmine could not be effective in fastening neuromuscular transmission recovery because TOF ratio is usually nearly zero at the time of skin incision. The drawback of sugammadex is the high cost approximately 100 USD per vial. To avoid postoperative residual neuromuscular block, sugammadex is not mandatory. Since merely single dose of NMBA is administrated, adequate neuromuscular transmission recovery is usually observed at the end of surgery. Therefore, neostigmine is probably fast enough in day-to-day anesthesia practice for conventional group.

Post-operaitve adverse events may have an impact on patient experience to surgery and anesthesia. To mitigate postoperative nausea, vomiting and pain, an anesthesia regimen was recommended to combine dexamethasone, propofol target-controlled infusion, parecoxib or NSAID and avoid postoperative opioids such as morphine. ${ }^{12}$

The current investigated protocol has several limitations. Firstly, only a small number of piglets were used. A wellestablished porcine model was used in the study. This widely applied protocol may enhance overall efficacy by following the replacement, reduction and refinement $(3 \mathrm{R})$ principles in IONM studies. ${ }^{22}$ We found that the results were highly reproducible in this study, hence, we believed that the data from the small number of piglets was sufficient. Secondly, the clinical trial was a retrospective study rather than a prospective, randomized study. The physical status of population in this trial was relatively healthy and patients with severe comorbidities were not enrolled in the study. The mean weight of all operated patients is about $64 \mathrm{~kg}$. The reversal time of NMB with sugammadex in obese or morbidly obese is still matter of debate. Furthermore, the use of sugammadex is not recommended in renal failure patients even with dialysis. We did not include extreme elderly and pediatric population which may affect reversal time. Hence, our result demonstrates a feasible protocol only for non-obese adult population without renal failure. Thirdly, we focus on titrating neuromuscular blocking agent to optimize neural monitoring as well as anesthesia protocol, we did not analyze the impact of this protocol on mapping of the abnormal situations in RLN or EBSLN. However, our result showed that the precise NMB management ensured timely neuromuscular function restoration for high-quality EMG signal. Thus, the benefit and efficiency of precise NMB management on facilitating neural mapping of the abnormal situations in RLN or EBSLN deserve further investigation. Finally, concerning expenditure in healthcare system, protocol including sugammadex is a high-cost therapy and of restrictive use in most countries currently. Conversely, a less expensive and ultra-short acting NMBAsuccinylcholine remains a choice for neural monitoring anesthesia. The only clinical caution is its rare side effects such as trismus, bronchospasm and malignant hyperthermia.

In conclusion, both porcine model and clinical application showed that precision NMB management by low-dose sugammadex was effective for IONM. There are some major advantages of precision $\mathrm{NMB}$ management for monitored thyroidectomy. Firstly, it allowed for adequate rocuronium dose to induce excellent tracheal intubation condition for EMG tube placement. Secondly, though low-dose sugamma$\operatorname{dex}(0.5 \mathrm{mg} / \mathrm{kg})$ only provided only partial neuromuscular function restoration, it still facilitated high-quality IONM $\left(\mathrm{V}_{1}\right)$ signal in a timely manner. Finally, a separate dose of sugammadex $(1.5 \mathrm{mg} / \mathrm{kg})$ at the end of surgery ensured safe and rapid extubation by complete neuromuscular function recovery. Therefore, we suggest including sugammadex administration into anesthesia strategy for IONM protocol to meet both anesthesia and surgery demands.

\section{Acknowledgments}

The authors express gratitude to Hsiu-Ya Chen (nurse anesthetist), Hui-Chun Chen (clinical nurse specialist), and Pao-Chu Hun (veterinarian, Laboratory Animal Center) for their excellent technical assistance.

\section{Disclosure statement}

The authors have no financial interests or conflicts of interest to declare. 


\section{Funding}

This study was supported by grants from the Kaohsiung Municipal Hsiao-Kang Hospital (Kmhk-108-027), the Ministry of Science and Technology, Taiwan (MOST 107-2314-B-037-113) and the Kaohsiung Medical University Hospital (KMUH107-7R89).

\section{ORCID}

Gianlorenzo Dionigi (D) http://orcid.org/0000-0003-0864-6087 Che-Wei Wu (iD) http://orcid.org/0000-0003-1052-5348

\section{References}

1. Randolph GW, Dralle $\mathrm{H}$, Abdullah $\mathrm{H}$, International Intraoperative Monitoring Study Group, et al. Electrophysiologic recurrent laryngeal nerve monitoring during thyroid and parathyroid surgery: international standards guideline statement. Laryngoscope. 2011;121 (Suppl 1):S1-S16. doi:10.1002/lary.21119.

2. Barczyński M, Randolph GW, Cernea CR, International Neural Monitoring Study Group, et al. External branch of the superior laryngeal nerve monitoring during thyroid and parathyroid surgery: International Neural Monitoring Study Group standards guideline statement. Laryngoscope. 2013;123 (Suppl 4):S1-S14. doi:10.1002/lary.24301.

3. Schneider R, Randolph GW, Dionigi G, et al. International neural monitoring study group guideline 2018 part I: staging bilateral thyroid surgery with monitoring loss of signal. Laryngoscope. 2018;128 (Suppl 3):S1-s17. doi:10.1002/lary.24301.

4. Wu CW, Dionigi G, Barczynski M, et al. International neuromonitoring study group guidelines 2018: Part II: optimal recurrent laryngeal nerve management for invasive thyroid cancer-incorporation of surgical, laryngeal, and neural electrophysiologic data. Laryngoscope. 2018;128 (Suppl 3):S18-S27. doi:10.1002/lary. 27360.

5. Zhang D, Wang T, Zhao Y, et al. Pre-prototype stimulating and recording endotracheal tube for continuous monitoring of the recurrent laryngeal nerve during thyroid surgery. J Investig Surg. Q3 2020;:1-11.

6. Ji YB, Ko SH, Song CM, et al. Feasibility and efficacy of intraoperative neural monitoring in remote access robotic and endoscopic thyroidectomy. Oral Oncol. 2020;103:104617. doi:10.1016/ j.oraloncology.2020.104617.

7. Barczynski M. A novel approach to continuous monitoring of the recurrent laryngeal nerve during thyroid surgery. Journal of Investigative Surgery: The Official Journal of the Academy of

Q4 $\begin{aligned} & \text { Surgical Research. 2020;:1-2. } \\ & \text { 8. Dionigi G, Wu CW, Kim HY, Rausei S, Boni L, Chiang FY. }\end{aligned}$ Severity of recurrent laryngeal nerve injuries in thyroid surgery. World J Surg. 2016;40(6):1373-1381. doi:10.1007/s00268-0163415-3.

9. Gurleyik E, Gurleyik G. Intraoperative monitoring of external branch of the superior laryngeal nerve: functional identification, motor integrity, and its role on vocal cord function. J Invest Surg. 2018;31(6):509-514. doi:10.1080/08941939.2017.1362489.

10. Wu CW, Lee KD, Tae K, et al. Recurrent laryngeal nerve (RLN) injury in thyroid surgery: lessons learned from the intraoperative neural monitoring (IONM). International Journal of Head and Neck Science. 2017;1(1):19-26.

11. $\mathrm{Wu} \mathrm{CW}$. Investigation the role of thyroid isthmusectomy for solitary isthmic papillary thyroid carcinoma. Journal of Investigative Surgery: The Official Journal of the Academy of Surgical Research. 2020;:1-2.

12. Lu IC, Lin IH, Wu CW, et al. Preoperative, intraoperative and postoperative anesthetic prospective for thyroid surgery: what's new. Gland Surg. 2017;6(5):469-475. doi:10.21037/gs.2017.05.02.
13. Han YD, Liang F, Chen P. Dosage effect of rocuronium on 764 intraoperative neuromonitoring in patients undergoing thyroid 765 surgery. Cell Biochem Biophys. 2015;71(1):143-146. doi:10.1007/ 766 s12013-014-0176-1.

14. Chang PY, Wu CW, Chen HY, et al. Influence of intravenous 767 anesthetics on neuromonitoring of the recurrent laryngeal nerve 768 during thyroid surgery. Kaohsiung J Med Sci. 2014;30(10): 769 499-503. doi:10.1016/j.kjms.2014.05.009.

15. Lu IC, Chu KS, Tsai CJ, et al. Optimal depth of NIM EMG 771 endotracheal tube for intraoperative neuromonitoring of the 772 recurrent laryngeal nerve during thyroidectomy. World J Surg. 772
2008;32(9):1935-1939. doi:10.1007/s00268-008-9549-1.

16. Stefanov CS, Dimov RS, Doikov IY, Konsulov SS. Method of 774 anaesthesia in thyroid surgery with intraoperative monitoring of 775 the recurrent laryngeal nerves. Folia Med (Plovdiv)). 2001;43(4): 776 $14-18$.

17. Lu IC, Wu CW, Chang PY, et al. Reversal of rocuronium- 778 induced neuromuscular blockade by sugammadex allows for 778 optimization of neural monitoring of the recurrent laryngeal 779 nerve. Laryngoscope. 2016;126(4):1014-1019. doi:10.1002/lary. 780 25577.

18. Empis de Vendin O, Schmartz D, Brunaud L, Fuchs-Buder T. 782 Recurrent laryngeal nerve monitoring and rocuronium: A select- 783 ive Sugammadex reversal protocol. World J Surg. 2017;41(9): 784 2298-2303. doi:10.1007/s00268-017-4004-9.

19. Lu IC, Chang PY, Su MP, et al. The feasibility of sugammadex 785 for general anesthesia and facial nerve monitoring in patients 786 undergoing parotid surgery. Kaohsiung J Med Sci. 2017;33(8): 787 400-404. doi:10.1016/j.kjms.2017.06.002.

20. Brunaud L, Fuchs-Buder T. In reference to Reversal of rocuro- 789 nium-induced neuromuscular blockade by Sugammadex allows 789 for optimization of neural monitoring of the recurrent laryngeal 790 nerve. Laryngoscope. 2017;127(1):E50doi:10.1002/lary.25990.

21. Lu IC, Wu CW, Chang PY, et al. In response to Reversal of 792 rocuronium-induced neuromuscular blockade by sugammadex 793 allows for optimization of neural monitoring of the recurrent 794 laryngeal nerve. Laryngoscope. 2017;127(1):E51-E52. doi:10.1002/ 795 lary.26036.

22. Wu CW, Huang TY, Chen HC, et al. Intra-operative neural 796 monitoring of thyroid surgery in a porcine model. JoVE. 2019; 797 (144) doi:10.3791/57919.

23. Lu IC, Chang PY, Randolph GW, et al. Safety of high-current 799 stimulation for intermittent intraoperative neural monitoring in 800 thyroid surgery: a porcine model. Laryngoscope. 2018;128(9): 801 2206-2212. doi:10.1002/lary.27086.

24. Wu CW, Lu IC, Randolph GW, et al. Investigation of optimal 802 intensity and safety of electrical nerve stimulation during intrao- 803 perative neuromonitoring of the recurrent laryngeal nerve: a pro- 804 spective porcine model. Head Neck. 2010;32(10):1295-1301. doi: 805 10.1002/hed.21324.

25. Wu C-W, Randolph GW, Lu I-C, et al. Intraoperative neural 806 monitoring in thyroid surgery: lessons learned from animal stud- 807 ies. Gland Surg. 2016;5(5):473-480. doi:10.21037/gs.2016.09.06. 808

26. Wu C-W, Liu X, Barczyński M, et al. Optimal stimulation dur- 809 ing monitored thyroid surgery: EMG response characteristics in 810 a porcine model. The Laryngoscopes. 2017;127(4):998-1005. doi: 811 10.1002/lary.26141.

27. Chiang FY, Lu IC, Kuo WR, Lee KW, Chang NC, Wu CW. The 812 mechanism of recurrent laryngeal nerve injury during thyroid 813 surgery-the application of intraoperative neuromonitoring. 814 Surgery. 2008;143(6):743-749. doi:10.1016/j.surg.2008.02.006. 815

28. Chiang FY, Lee KW, Chen HC, et al. Standardization of intrao- 816 perative neuromonitoring of recurrent laryngeal nerve in thyroid 817 operation. World J Surg. 2010;34(2):223-229. doi:10.1007/s00268- 818 009-0316-8.

29. Lu IC, Tsai CJ, Wu CW, et al. A comparative study between 1819 and 2 effective doses of rocuronium for intraoperative neuromo- 820 nitoring during thyroid surgery. Surgery. 2011;149(4):543-548. 821 doi:10.1016/j.surg.2010.11.006. 


$$
e^{2^{0^{0^{2}}}}
$$

